\title{
ON THE CONVERGENCE OF ORDERED SETS OF PROJECTIONS ${ }^{1}$
}

\author{
JOHN Y. BARRY
}

E. R. Lorch has shown $[3$, p. 223] that a uniformly bounded, naturally ordered sequence of projections in a reflexive Banach space converges to its supremum in the strong topology of operators. In this note it is shown that a uniformly bounded, naturally ordered set of projections in a Banach space $X$ having a weak $x$-cluster point for each $x \in X$ converges to its supremum in the strong topology of operators. The method of proof is substantially different from that of Lorch.

A bounded operator $E$, acting in a Banach space $X$, is a projection if $E^{2}=E$. Letting $I$ denote the identity projection, $E(X)$ and $(I-E)(X)$ are strongly closed manifolds.

The projections in $X$ have a natural order: $E_{1} \leqq E_{2}$ if $E_{1} E_{2}=E_{2} E_{1}$ $=E_{1}$. If $\{\alpha\}$ is a directed set, and $\alpha_{1} \leqq \alpha_{2}$ implies $E_{\alpha_{1}} \leqq E_{\alpha_{2}}$, then $\left\{E_{\alpha}\right\}$ is naturally ordered.

If $Y$ is a subset of $X, w(Y)$ will denote the weak closure of $Y$. The span of $Y$, denoted by $\operatorname{sp}(Y)$, is the strongly closed linear manifold generated by $Y$.

Suppose $\left\{E_{\alpha}\right\}$ is a set of projections in $X$. A projection $E$ such that $E(X)=\operatorname{sp}\left(\cup_{\alpha} E_{\alpha}(X)\right)$ and $(I-E)(X)=\bigcap_{\alpha}\left(I-E_{\alpha}\right)(X)$ is the supremum of $\left\{E_{\alpha}\right\}$, and is denoted by $U_{\alpha} E_{\alpha}$.

If $\left\{E_{\alpha}\right\}$ is naturally ordered, $y_{x}$ is a weak $x$-cluster point of $\left\{E_{\alpha}\right\}$ if $y_{x} \in \bigcap_{\alpha} w\left(\left\{E_{\beta}(x) \mid \beta \geqq \alpha\right\}\right)$.

The central result of this note follows:

THEOREM 1. If $\left\{E_{\alpha}\right\}$ is a naturally ordered, uniformly bounded set of projections in $X$, then $\lim _{\alpha} E_{\alpha}=\bigcup_{\alpha} E_{\alpha}$ in the strong topology of operators if and only if $\left\{E_{\alpha}\right\}$ has a weak $x$-cluster point for each $x \in X$.

Proof. Sufficiency. Pick $x \in X$, and a projection $E_{\alpha_{0}}$. Let $y_{x}$ be a weak $x$-cluster point of $\left\{E_{\alpha}\right\}$. Put $N_{\epsilon}\left(y_{x}\right)=\left\{z||\left(E_{\alpha_{0}}^{*} x^{*}\right)\left(z-y_{x}\right) \mid<\epsilon\right\}$. By assumption, there is a $\beta \geqq \alpha_{0}$ such that $\epsilon>\left|E_{\alpha_{0}}^{*} x^{*}\left(E_{\beta}(x)-y_{x}\right)\right|$ $=\left|x^{*}\left(E_{\alpha_{0}}(x)-E_{\alpha_{0}}\left(y_{x}\right)\right)\right|$. Thus, $E_{\alpha_{0}}(x)=E_{\alpha_{0}}\left(y_{x}\right)$.

Since $y_{x} \in w\left(\left\{E_{\alpha}(x)\right\}\right)$, it follows by a theorem of Banach [2, p. 58] that $y_{x}=\lim _{n \rightarrow \infty} T_{n}(x)$, where $T_{n}=\sum_{k=1}^{n} c_{k}^{n} E_{\alpha_{k}}$.

Clearly, $\lim _{\alpha} E_{\alpha} T_{n}=T_{n}$ in the strong topology of operators. Furthermore,

Received by the editors August 3, 1953.

${ }^{1}$ Work sponsored by Office of Naval Research under contract ONR 609 (04). 


$$
\begin{aligned}
\left\|E_{\alpha}(x)-\left(E_{\alpha} T_{n}\right)(x)\right\| & =\left\|E_{\alpha}\left(y_{x}\right)-\left(E_{\alpha} T_{n}\right)(x)\right\| \\
& \leqq M\left\|y_{x}-T_{n}(x)\right\|,
\end{aligned}
$$

where $M=\sup _{\alpha}\left\|E_{\alpha}\right\|$. Thus, $\lim _{n \rightarrow \infty}\left(E_{\alpha} T_{n}\right)(x)=E_{\alpha}(x)$, uniformly in $\alpha$.

By the E. H. Moore theorem on the interchange of limits $[4, p$. 116],

$$
\begin{aligned}
y_{x} & =\lim _{n \rightarrow \infty} \lim _{\alpha}\left(E_{\alpha} T_{n}(x)=\lim _{\alpha} \lim _{n \rightarrow \infty}\left(E_{\alpha} T_{n}\right)(x)\right. \\
& =\lim _{\alpha} E_{\alpha}(x) .
\end{aligned}
$$

Put $E(x)=y_{x}$. It is now easily verified that $E$ is a projection in $X$. If $x \in \bigcap_{\alpha}\left(I-E_{\alpha}\right)(X)$, then $x=\lim _{\alpha} \quad\left(I-E_{\alpha}\right)(x)=(I-E)(x)$. Thus, $(I-E)(X) \supset \bigcap_{\alpha}\left(I-E_{\alpha}\right)(X)$. Since $E(x)=\lim _{\alpha} E_{\alpha}(x), E(X)$ $C \operatorname{sp}\left(\cup_{\alpha} E_{\alpha}(X)\right)$. However, noting $E E_{\alpha}=E_{\alpha}$, and $\left(I-E_{\alpha}\right)(I-E)$ $=I-E$, it is clear that $E(X) \supset \operatorname{sp}\left(\bigcup_{\alpha} E_{\alpha}(X)\right)$, and $(I-E)(X)$ $C \cap_{\alpha}\left(I-E_{\alpha}\right)(X)$. Therefore, $E=\bigcup_{\alpha} E_{\alpha}$.

Necessity is clear.

COROLlaRy 1. If $\left\{E_{\alpha}\right\}$ is a naturally ordered, uniformly bounded set of projections in $X$, and $\left\{E_{\alpha}(x)\right\}$ is weakly conditionally compact for each $x \in X$, then $\lim _{\alpha} E_{\alpha}=\cup_{\alpha} E_{\alpha}$ in the strong topology of operators.

Proof. Pick $x \in X$. If $\beta_{0} \geqq \alpha_{1}, \cdots, \alpha_{n}$, then

$$
E_{\beta_{0}}(x) \in \bigcup_{k=1}^{n} w\left(\left\{E_{\beta}(x) \mid \beta \geqq \alpha_{k}\right\}\right) .
$$

Consequently, $\cap_{\alpha} w\left(\left\{E_{\beta}(x) \mid \beta \geqq \alpha\right\}\right) \neq \varnothing$.

CoRollary 2. If $\left\{E_{\alpha}\right\}$ is a naturally ordered, uniformly bounded set of projections in a reflexive Banach space, then $\lim _{\alpha} E_{\alpha}=\cup_{\alpha} E_{\alpha}$ in the strong topology of operators.

Proof. Let $M=\sup _{\alpha}\left\|E_{\alpha}\right\|$, and $S(x)=\{z \mid\|z\| \leqq M\|x\|\}$. By a theorem of Alaoglu [1, p. 255], $S(x)$ is weakly compact. The desired result now follows from Corollary 1.

\section{REFERENCES}

1. L. Alaoglu, Weak topologies of normed linear spaces, Ann. of Math. vol. 41 (1940) pp. 252-267.

2. S. Banach, Théorie des opérations linéaires, Warsaw, 1932.

3. E. R. Lorch, On a calculus of operators in reflexive vector spaces, Trans. Amer. Math. Soc. vol. 45 (1939) pp. 217-234.

4. E. H. Moore and H. L. Smith, A general theory of limits, Amer. J. Math. vol. 44 (1922) pp. 102-121.

YALE UNIVERSITY 